\title{
Compound Means and Fast Computation of Radicals
}

\author{
Jan Šustek \\ Department of Mathematics, Faculty of Science, University of Ostrava, Ostrava, Czech Republic \\ Email: jan.sustek@osu.cz
}

Received 28 June 2014; revised 2 August 2014; accepted 16 August 2014

Copyright (C) 2014 by author and Scientific Research Publishing Inc.

This work is licensed under the Creative Commons Attribution International License (CC BY).

http://creativecommons.org/licenses/by/4.0/

(c) (i) Open Access

\begin{abstract}
In last decades, several algorithms were developed for fast evaluation of some elementary functions with very large arguments, for example for multiplication of million-digit integers. The present paper introduces a new fast iterative method for computing values $\sqrt[k]{x}$ with high accuracy, for fixed $k \in \mathbb{N}$ and $x \in \mathbb{R}^{+}$. The method is based on compound means and Padé approximations.
\end{abstract}

Keywords

Compound Means, Padé Approximation, Computation of Radicals, Iteration

\section{Introduction}

In last decades, several algorithms were developed for fast evaluation of some elementary functions with very large arguments, for example for multiplication of million-digit integers. The present paper introduces a new iterative method for computing values $\sqrt[k]{x}$ with high accuracy, for fixed $k \in \mathbb{N}$ and $x \in \mathbb{R}^{+}$.

The best-known method used for computing radicals is Newton's method used to solve the equation

$$
f(t)=t^{k}-x=0 .
$$

Newton's method is a general method for numerical solution of equations and for particular choice of the equation it can lead to useful algorithms, for example to algorithm for division of long numbers. This method converges quadratically. Householder [1] found a generalization of this method. Let $d \in \mathbb{N}_{0}$ be a parameter of the method. When solving the equation $f(t)=0$ the iterations converging to the solution are 


$$
a_{n+1}=a_{n}+(d+1) \frac{\left(\frac{1}{f}\right)^{(d)}\left(a_{n}\right)}{\left(\frac{1}{f}\right)^{(d+1)}\left(a_{n}\right)} .
$$

The convergence has order $d+2$. The order of convergence can be made arbitrarily large by the choice of $d$. But for larger values of $d$ it is necessary to perform too many operations in every step and the method gets slower.

The method (3.4) presented in this paper involves compound means. It is proved that this method performs less operations and is faster than the former methods.

Definition 1. A function $P: \mathbb{R}^{+} \times \mathbb{R}^{+} \rightarrow \mathbb{R}^{+}$is called mean if for every $t, u \in \mathbb{R}^{+}$

$$
\min (t, u) \leq P(t, u) \leq \max (t, u) .
$$

A mean $P$ is called strict if

$$
t \neq u \Rightarrow \min (t, u)<P(t, u)<\max (t, u) .
$$

A mean $P$ is called continuous if the function $P$ is continuous.

A known class of means is the power means defined for $p \neq 0$ by

$$
M_{p}(t, u):=\left(\frac{t^{p}+u^{p}}{2}\right)^{\frac{1}{p}} ;
$$

for $p=0$ we define

$$
M_{0}(t, u):=\lim _{p \rightarrow 0} M_{p}(t, u)=\sqrt{t u} .
$$

The most used power means are the arithmetic mean $A=M_{1}$, the geometric mean $G=M_{0}$ and the harmonic mean $H=M_{-1}$. All power means are continuous and strict. There is a known inequality between power means

$$
\alpha \leq \beta \Rightarrow M_{\alpha} \leq M_{\beta},
$$

see e.g. [2]. From this one directly gets the inequality between arithmetic mean and geometric mean. For other classes of means see e.g. [3].

Taking two means, one can obtain another mean by composing them by the following procedure.

Definition 2. Let $P, Q$ be two means. Given two positive numbers $t, u$, put

$$
\begin{gathered}
a_{0}:=t, \quad b_{0}:=u, \\
a_{n+1}:=P\left(a_{n}, b_{n}\right), \quad b_{n+1}:=Q\left(a_{n}, b_{n}\right),
\end{gathered}
$$

$n \geq 0$. If these two sequences converge to a common limit then this limit is denoted by

$$
P \diamond Q(t, u):=\lim _{n \rightarrow \infty} a_{n}=\lim _{n \rightarrow \infty} b_{n} .
$$

The function $P \diamond Q: \mathbb{R}^{+} \times \mathbb{R}^{+} \rightarrow \mathbb{R}^{+}$is called compound mean.

The best known application of compound means is Gauss' arithmetic-geometric mean [4]

$$
A \diamond G(t, u)=\frac{\pi}{2 \int_{0}^{\pi / 2} \frac{\mathrm{d} \theta}{\sqrt{t^{2} \cos ^{2} \theta+u^{2} \sin ^{2} \theta}} .} .
$$

Iterations of the compound mean then give a fast numerical algorithm for computation of the elliptic integral (1.2).

Matkowski [5] proved the following theorem on existence of compound means.

Theorem 1. Let $P, Q$ be continuous means such that at least one of them is strict. Then the compound mean $P \diamond Q$ exists and is continuous. 


\section{Properties}

We call a mean $P$ homogeneous if for every $c, t, u \in \mathbb{R}^{+}$

$$
P(c t, c u)=c P(t, u) .
$$

All power means are homogeneous. If two means are homogeneous then their compound mean is also homogeneous.

Homogeneous mean $P$ can be represented by its trace

$$
p(t)=P(1, t) .
$$

Conversely, every function $p: \mathbb{R}^{+} \rightarrow \mathbb{R}^{+}$with property

$$
\min (1, t) \leq p(t) \leq \max (1, t)
$$

represents homogeneous mean

$$
P(t, u)=t p\left(\frac{u}{t}\right)
$$

Theorem 2. If the compound mean $P \diamond Q$ exists then it satisfies the functional equation

$$
P \diamond Q(P(t, u), Q(t, u))=P \diamond Q(t, u) .
$$

On the other hand, there is only one mean $R$ satisfying the functional equation

$$
R(P(t, u), Q(t, u))=R(t, u) .
$$

Easy proofs of these facts can be found in [5].

Example 1. Take the arithmetical mean $A(t, u)=\frac{t+u}{2}$ and the harmonic mean $H(t, u)=\frac{2 t u}{t+u}$. The arithmetic-harmonic mean $A \diamond H$ exists by Theorem 1 and Theorem 2 implies that $A \diamond H=G$. Hence the iterations of the arithmetic-harmonic mean $A \diamond H(1, x)$ can be used as a fast numerical method of computation of $G(1, x)=\sqrt{x}$. This leads to a well known Babylonian method

$$
a_{0}=1, \quad a_{n+1}=\frac{1}{2}\left(a_{n}+\frac{x}{a_{n}}\right)
$$

with a quadratical convergence to $\lim _{n \rightarrow \infty} a_{n}=\sqrt{x}$.

The Babylonian method is in fact Newton's method used to solve the equation $t^{2}-x=0$. Using Newton's method to solve the equation $t^{k}-x=0$ leads to iterations

$$
a_{0}=1, \quad a_{n+1}=\frac{1}{k}\left((k-1) a_{n}+\frac{x}{a_{n}^{k-1}}\right)
$$

with a quadratical convergence to $\lim _{n \rightarrow \infty} a_{n}=\sqrt[k]{x}$.

\section{Our Method}

In the present paper we will proceed similarly as in Example 1. For a fixed integer $k \geq 2$ and a positive real number $x$ we will find a sequence of approximations converging fast to $\sqrt[k]{x}$.

We need the following lemma.

Lemma 1. Let function $p$ satisfy $p^{(j)}(1)=(\sqrt[k]{ })^{(j)}(1)$ for $j=0, \cdots, w$ and let $p^{(w+1)}$ be bounded. Let $q(t)=\frac{t}{p^{k-1}(t)}$. Assume that $p$ and $q$ satisfy (2.1) strictly if $t \neq 1$. Then the function $q$ satisfies $q^{(j)}(1)=p^{(j)}(1)$ for $j=0, \cdots, w$ and $q^{(w+1)}$ is bounded. Let $P$ and $Q$ be the homogeneous means corresponding to traces $p$ and $q$, respectively. Then the compound mean $P \diamond Q$ exists and its convergence has 
order $w+1$.

Proof. The assumption implies that

$$
p(t)=\sqrt[k]{t}+O(t-1)^{w+1}
$$

Then

$$
\begin{aligned}
q(t) & =\frac{t}{p^{k-1}(t)}=\frac{t}{\left(\sqrt[k]{t}+O(t-1)^{w+1}\right)^{k-1}} \\
& =\frac{t}{t^{\frac{k-1}{k}}+O(t-1)^{w+1}}=\sqrt[k]{t}+O(t-1)^{w+1}
\end{aligned}
$$

hence $q^{(j)}(1)=p^{(j)}(1)$ for $j=0, \cdots, w$ and $q^{(w+1)}$ is bounded.

The compound mean $P \diamond Q$ exists by Theorem 1. Let $a_{n}$ and $b_{n}$ be the iterations of $P \diamond Q$. To find the order of convergence put $\delta_{n}:=\frac{b_{n}-a_{n}}{a_{n}}$. Then

$$
\begin{aligned}
& a_{n+1}=p\left(a_{n}, a_{n}\left(1+\delta_{n}\right)\right)=a_{n}\left(1+\delta_{n}\right)=a_{n} \sum_{j=0}^{w} \frac{p^{(j)}(1)}{j !} \delta_{n}^{j}+O\left(\delta_{n}^{w+1}\right), \\
& b_{n+1}=p\left(a_{n}, a_{n}\left(1+\delta_{n}\right)\right)=a_{n}\left(1+\delta_{n}\right)=a_{n} \sum_{j=0}^{w} \frac{q^{(j)}(1)}{j !} \delta_{n}^{j}+O\left(\delta_{n}^{w+1}\right)
\end{aligned}
$$

and $b_{n+1}-a_{n+1}=O\left(\delta_{n}^{w+1}\right)=O\left(b_{n}-a_{n}\right)^{w+1}$.

Take the mean $R_{k}(t, u):=\sqrt[k]{t^{k-1} u}$. This mean is strict, continuous and homogeneous and it has the property $R_{k}(1, x)=\sqrt[k]{x}$. We will construct two means $P, Q$ such that $R_{k}=P \diamond Q$.

Let $s \in \mathbb{N}$ and let $p(t)$ be the Padé approximation of the function $\sqrt[k]{t}$ of order $[s, s]$ around $t=1$,

$$
p(t)=\frac{\sum_{j=0}^{s} e_{j} t^{j}}{\sum_{j=0}^{s} f_{j} t^{j}}=\sqrt[k]{t}+O(t-1)^{2 s+1}
$$

The exact formula for $e_{j}, f_{j}$ will be derived in Lemma 15. In Lemma 20 we will prove that $p$ satisfies

$$
\min (1, \sqrt[k]{t})<p(t)<\max (1, \sqrt[k]{t})
$$

for every $t \in \mathbb{R}^{+}$. Hence it is a trace of a strict homogeneous mean

$$
P(t, u)=t p\left(\frac{u}{t}\right)=t \frac{\sum_{j=0}^{s} e_{j}\left(\frac{u}{t}\right)^{j}}{\sum_{j=0}^{s} f_{j}\left(\frac{u}{t}\right)^{j}} .
$$

Relation $R_{k}=P \diamond Q$ and Theorem 2 imply that

and its trace is

$$
Q(t, u)=\frac{t^{k-1} u}{P^{k-1}(t, u)}
$$

$$
q(t)=Q(1, t)=\frac{t}{p^{k-1}(t)}
$$

Inequalities (3.2) imply that $Q$ is a strict homogeneous mean too.

As in Definition 2, denote the sequences given by the compound mean $P \diamond Q$ by $a_{n}, b_{n}$, starting with 
$a_{0}=1$ and $b_{0}=x$. From (3.3) we obtain that

$$
b_{n+1}=Q\left(a_{n}, b_{n}\right)=\frac{a_{n}^{k-1} b_{n}}{P^{k-1}\left(a_{n}, b_{n}\right)}=\frac{a_{n}^{k-1} b_{n}}{a_{n+1}^{k-1}},
$$

hence $a_{n+1}^{k-1} b_{n+1}=a_{n}^{k-1} b_{n}=\cdots=a_{0}^{k-1} b_{0}=x$ and $b_{n}=\frac{x}{a_{n}^{k-1}}$. So the iterations of the compound mean $P \diamond Q$ are

$$
a_{n+1}=P\left(a_{n}, b_{n}\right)=P\left(a_{n}, \frac{x}{a_{n}^{k-1}}\right)=a_{n} \frac{\sum_{j=0}^{s} e_{j}\left(\frac{x}{a_{n}^{k}}\right)^{j}}{\sum_{j=0}^{s} f_{j}\left(\frac{x}{a_{n}^{k}}\right)^{j}}=a_{n} \frac{\sum_{j=0}^{s} e_{j} x^{j}\left(a_{n}^{k}\right)^{s-j}}{\sum_{j=0}^{s} f_{j} x^{j}\left(a_{n}^{k}\right)^{s-j}} .
$$

Note that we don't have to compute the sequence $b_{n}$.

According to (3.1) and Lemma 1 the convergence of the sequence (3.4) to its limit $\sqrt[k]{x}$ has order $2 s+1$.

\section{Complexity}

Let $M(N)$ denote the time complexity of multiplication of two $N$-digit numbers. The classical algorithm of multiplication has asymptotic complexity $M(N)=O\left(N^{2}\right)$. But there are also algorithms with asymptotic complexity

$$
\begin{gathered}
M(N)=O\left(N^{\log _{2} 3}\right)=O\left(N^{1.585}\right), \\
M(N)=O(N \log N \log \log N)
\end{gathered}
$$

or

$$
M(N)=O\left(N \log N 2^{\log ^{*} N}\right),
$$

see Karatsuba [6], Schönhage and Strassen [7] or Fürer [8], respectively. The fastest algorithms have large asymptotic constants, hence it is better to use the former algorithms if the number $N$ is not very large.

The complexity of division of two $\mathrm{N}$-digit numbers differs from the complexity of multiplication only by some multiplicative constant $D$. Hence the complexity of division is $D M(N)$. Analysis in [9] shows that this constant can be as small as $D=\frac{7}{2}$.

We will denote by $\sigma(k)$ the minimal number of multiplications necessary to compute the power $t \mapsto t^{k}$. See [10] for a survey on known results about the function $\sigma$.

Before the main computation of complexity we need this auxiliary lemma.

Lemma 2. Assume that $r \in \mathbb{N}$ and that $M(N)$ is a function such that for some $\omega \in[1,2]$ the function $f(N)=\frac{M(N)}{N^{\omega}}$ is nondecreasing with

$$
f(N)=N^{o(1)}
$$

For every $\delta>0$ put $g_{\delta}(N)=\frac{N^{\omega+\delta}}{M(N)}$ and assume that

1) for every $T$ the image set $g_{\delta}([1, T])$ is bounded and

2) there is $N_{0}(\delta)$ with $g_{\delta}(n) \geq g_{\delta}(m)$ for every $n \geq m \geq N_{0}(\delta)$.

Then

$$
\lim _{N \rightarrow \infty} \frac{\sum_{j=1}^{\left\lfloor\log _{r} N\right\rfloor} M\left(\frac{N}{r^{j}}\right)}{M(N)}=\frac{1}{r^{\omega}-1} .
$$


Proof. From the monotonicity of $f$ we have for every $N$

$$
\frac{\sum_{j=1}^{\left\lfloor\log _{r} N\right\rfloor} M\left(\frac{N}{r^{j}}\right)}{M(N)}=\frac{\sum_{j=1}^{\left\lfloor\log _{r} N\right\rfloor} \frac{N^{\omega}}{r^{\omega j}} f\left(\frac{N}{r^{j}}\right)}{N^{\omega} f(N)} \leq \sum_{j=1}^{\left\lfloor\log _{r} N\right\rfloor} \frac{1}{r^{\omega j}} \leq \frac{1}{r^{\omega}-1} .
$$

Let $\delta>0$. Then (4.1) implies $\lim _{N \rightarrow \infty} g_{\delta}(N)=\infty$. From this and properties 1 and 2 we deduce that there exists a number $N_{1}(\delta)$ such that $g_{\delta}(n) \geq g_{\delta}(m)$ for every $n \geq N_{1}(\delta)$ and every $m \leq n$. This implies for every $N \geq N_{1}(\delta)$

$$
\frac{\sum_{j=1}^{\left\lfloor\log _{r} N\right\rfloor} M\left(\frac{N}{r^{j}}\right)}{M(N)}=\frac{\sum_{j=1}^{\left\lfloor\log _{r} N\right\rfloor} \frac{N^{\omega+\delta}}{r^{(\omega+\delta) j} g_{\delta}\left(\frac{N}{r^{j}}\right)}}{\frac{N^{\omega+\delta}}{g_{\delta}(N)}} \geq \sum_{j=1}^{\left\lfloor\log _{r} N\right\rfloor} \frac{1}{r^{(\omega+\delta) j}}=\frac{1-\frac{1}{r^{(\omega+\delta)\left\lfloor\log _{r} N\right\rfloor}}}{r^{(\omega+\delta}-1} .
$$

Inequalities (4.2) and (4.3) yield

$$
\frac{1}{r^{\omega+\delta}-1} \leq \liminf _{N \rightarrow \infty} \frac{\sum_{j=1}^{\left\lfloor\log _{r} N\right\rfloor} M\left(\frac{N}{r^{j}}\right)}{M(N)} \leq \limsup _{N \rightarrow \infty} \frac{\sum_{j=1}^{\left\lfloor\log _{r} N\right\rfloor} M\left(\frac{N}{r^{j}}\right)}{M(N)} \leq \frac{1}{r^{\omega}-1} .
$$

Passing to the limit $\delta \rightarrow 0$ implies the result.

Note that all the above mentioned functions $M(N)$ satisfy all assumptions of Lemma 2 with $\omega=2$, $\omega=\log _{2} 3, \omega=1$ and $\omega=1$, respectively.

Now we compute the complexity $V(N)$ of algorithm computing $\sqrt[k]{x}$ to within $N$ digits. The functions $M(N)$ and $V(N)$ have asymptotically the same order as $N \rightarrow \infty$, see for instance Theorem 6.3 in [3]. Hence all fast algorithms for computing $\sqrt[k]{x}$ differ only in the asymptotic constants.

Let the algorithm for computing $\sqrt[k]{x}$

- performs $Z$ multiplications of two $N$-digit numbers before the iterations,

- performs $A$ multiplications and $B$ divisions of two long numbers in every step,

- has order of convergence $r$.

The accuracy to within $N$ digits is necessary only in the last step. In the previous step we need accuracy only to within $\frac{N}{r}$ digits and so on. Hence

$$
V(N)=V\left(\frac{N}{r}\right)+(A+B D) M(N)+O(N) .
$$

The error term $O(N)$ corresponds to additions of $N$-digit numbers. This and Lemma 2 imply that ${ }^{1}$

$$
\begin{aligned}
V(N) & =(A+B D) \sum_{j=0}^{\left\lfloor\log _{r} N\right\rfloor} M\left(\frac{N}{r^{j}}\right)+Z M(N)+O(N) \\
& =\left(\left(1+\frac{1}{r^{\omega}-1}+o(1)\right)(A+B D)+Z\right) M(N)+O(N) \\
& =\left(\left(1+\frac{1}{r^{\omega}-1}\right)(A+B D)+Z+o(1)\right) M(N) .
\end{aligned}
$$

\subsection{Complexity of Newton's Method}

Newton's method (2.3) has order 2 and in every step it performs $\sigma(k-1)$ multiplications (evaluation of $a_{n}^{k-1}$ )

${ }^{1}$ In the last line we assume the hypothesis that all multiplication algorithms satisfy $\frac{N}{M(N)}=o(1)$, see [12]. 
and 1 division. So the complexity is

$$
V^{\mathrm{N}}(k, N)=\left(S^{\mathrm{N}}(k)+o(1)\right) M(N)
$$

where

$$
S^{\mathrm{N}}(k)=\left(1+\frac{1}{2^{\omega}-1}\right)(\sigma(k-1)+D) \text {. }
$$

For the choice of multiplication and division algorithms with $\omega=1$ and $D=\frac{7}{2}$ we have

$$
V^{\mathrm{N}}(k, N)=(2 \sigma(k-1)+7+o(1)) M(N) .
$$

\subsection{Complexity of Householder's Method}

Consider Householder's method (1.1) applied to the equation $f(t)=t^{k}-x=0$. Let $d \in \mathbb{N}_{0}$. An easy calculation (see [11] for instance) leads to iterations

$$
a_{n+1}=a_{n} \frac{\sum_{j=0}^{d} \lambda_{j} x^{j}\left(a_{n}^{k}\right)^{d-j}}{\sum_{j=0}^{d} \mu_{j} x^{j}\left(a_{n}^{k}\right)^{d-j}} .
$$

where $\lambda_{j}(k, d)$ and $\mu_{j}(k, d)$ are suitable constants. The method has order $d+2$, before the iterations it performs $d-1$ multiplications of $N$-digit numbers (evaluation of $x^{2}, \cdots, x^{d}$ ) and in every step it performs $\sigma(k)+2 d-1$ multiplications (evaluation of $a_{n}^{k}$, then evaluations of numerator and denominator by Horner's method, and then the final multiplication) and 1 division. So the complexity of Householder's algorithm is

$$
V_{d}^{\mathrm{H}}(k, N)=\left(S_{d}^{\mathrm{H}}(k)+o(1)\right) M(N)
$$

where

$$
S_{d}^{\mathrm{H}}(k)=\left(1+\frac{1}{(d+2)^{\omega}-1}\right)(\sigma(k)+2 d-1+D)+d-1 .
$$

For the choice of multiplication and division algorithms with $\omega=1$ and $D=\frac{7}{2}$ we have

$$
V_{d}^{\mathrm{H}}(k, N)=\left(3 d+\sigma(k)+\frac{7}{2}+\frac{2 \sigma(k)+1}{2 d+2}+o(1)\right) M(N) .
$$

The optimal value of $d$ which minimizes the complexity is in this case

$$
d \in\left\{\left[\sqrt{\frac{2 \sigma(k)+1}{6}}-1\right],\left[\sqrt{\frac{2 \sigma(k)+1}{6}}-1\right]\right\} .
$$

\subsection{Complexity of Our Method}

Given $s \in \mathbb{N}$, our method (3.4) has order $2 s+1$, before the iterations it performs $s-1$ multiplications of $N$-digit numbers (evaluation of $x^{2}, \cdots, x^{s}$ ) and in every step it performs $\sigma(k)+2 s-1$ multiplications (evaluation of $a_{n}^{k}$, then evaluations of numerator and denominator by Horner's method ${ }^{2}$, and then the final multiplication) and 1 division. So the complexity of our algorithm is

$$
V_{s}^{\mathrm{S}}(k, N)=\left(S_{s}^{\mathrm{S}}(k)+o(1)\right) M(N)
$$

where

${ }^{2}$ Not always Horner's method is optimal, see [13]. In those cases Householder's and our algorithms are faster. 


$$
S_{s}^{\mathrm{S}}(k)=\left(1+\frac{1}{(2 s+1)^{\omega}-1}\right)(\sigma(k)+2 s-1+D)+s-1
$$

For the choice of multiplication and division algorithms with $\omega=1$ and $D=\frac{7}{2}$ we have

$$
V_{s}^{S}(k, N)=\left(3 s+\sigma(k)+\frac{5}{2}+\frac{2 \sigma(k)+5}{4 s}+o(1)\right) M(N) .
$$

The optimal value of $s$ which minimizes the complexity is in this case

$$
\left.s \in\left\{\mid \sqrt{\frac{2 \sigma(k)+5}{12}}\right\rfloor,\left|\sqrt{\frac{2 \sigma(k)+5}{12}}\right|\right\} .
$$

\section{Examples}

Example 2. Compare the algorithms for computation of $\sqrt[14]{x}$. For $k=14$ we have $\sigma(k)=14$ and, according to (4.4), the optimal value of $s$ for our algorithm is $s=1$. Padé approximation of the function $\sqrt[14]{t}$ around $t=1$ is

$$
\sqrt[14]{t}=\frac{15 t+13}{13 t+15}+O(t-1)^{3}
$$

Hence the iterations of our algorithm are

$$
a_{0}:=1, \quad a_{n+1}:=a_{n} \frac{15 x+13 a_{n}^{14}}{13 x+15 a_{n}^{14}}
$$

with convergence of order 3. For computation of $N$ digits of $\sqrt[14]{x}$ we need to perform

operations.

$$
V_{1}^{\mathrm{S}}(14, N)=\left(\frac{57}{4}+o(1)\right) M(N)
$$

Newton's method

$$
a_{0}:=1, \quad a_{n+1}:=\frac{1}{14}\left(13 a_{n}+\frac{x}{a_{n}^{13}}\right)
$$

has order 2 and for computation of $N$ digits it needs $(\sigma(13)=5)$

$$
V^{\mathrm{N}}(14, N)=(17+o(1)) M(N)
$$

operations. Hence our method saves $16 \%$ of time compared to Newton's method.

For Householder's method the optimal value is $d=1$ and it leads to the same iterations (5.1) as our method.

Example 3. Compare the algorithms for computation of $\sqrt[179]{x}$. For $k=179$ we have $\sigma(k)=10$ and, according to (4.4), the optimal value of $s$ for our algorithm is $s=2$. Padé approximation of the function $\sqrt[179]{t}$ around $t=1$ is

$$
\sqrt[179]{t}=\frac{10770 t^{2}+42721 t+10591}{10591 t^{2}+42721 t+10770}+O(t-1)^{5}
$$

Hence the iterations of our algorithm are

$$
a_{0}:=1, \quad a_{n+1}:=a_{n} \frac{10770 x^{2}+42721 x a_{n}^{179}+10591 a_{n}^{358}}{10591 x^{2}+42721 x a_{n}^{179}+10770 a_{n}^{358}}
$$

with convergence of order 5 . For computation of $N$ digits of $\sqrt[179]{x}$ we need to perform

$$
V_{2}^{\mathrm{S}}(179, N)=\left(\frac{173}{8}+o(1)\right) M(N)
$$


operations.

Newton's method

$$
a_{0}:=1, \quad a_{n+1}:=\frac{1}{179}\left(178 a_{n}+\frac{x}{a_{n}^{178}}\right)
$$

has order 2 and for computation of $N$ digits it needs

$$
V^{\mathrm{N}}(179, N)=(27+o(1)) M(N)
$$

operations.

For Householder's method the optimal value is $d=1$ and it leads to iterations

$$
a_{0}:=1, \quad a_{n+1}:=a_{n} \frac{180 x+178 a_{n}^{179}}{178 x+180 a_{n}^{179}} .
$$

This method has order 3 and for computation of $N$ digits it needs

$$
V_{1}^{\mathrm{H}}(179, N)=\left(\frac{87}{4}+o(1)\right) M(N)
$$

operations.

Hence our method saves $20 \%$ of time compared to Newton's method and saves $0.6 \%$ of time compared to Householder's method.

Example 4. Compare the algorithms for computation of $\sqrt[1234567890133]{x}$. For $k=1234567890133$ the exact value of $\sigma(k)$ is not known. We assume that $\sigma(k)=59$. According to (4.4), the optimal value of $s$ for our algorithm is $s=3$. The iterations of our algorithm are

$$
\begin{aligned}
& a_{0}:=1 \text {, } \\
& a_{n+1}:=a_{n}\left(\begin{array}{l}
94083818620004407126666191157329760 x^{3} \\
+846754367579125169414786634836768160 x^{2} a_{n}^{1234567890133} \\
+846754367578439298370880283615327200 x a_{n}^{2469135780266} \\
+94083818619724978182852492511557517 a_{n}^{3703703670399} \\
94083818619724978182852492511557517 x^{3} \\
+846754367578439298370880283615327200 x^{2} a_{n}^{1234567890133} \\
+846754367579125169414786634836768160 x a_{n}^{2469135780266} \\
+94083818620004407126666191157329760 a_{n}^{3703703670399}
\end{array}\right)
\end{aligned}
$$

with convergence of order 7. For computation of $N$ digits of $\sqrt[1234567890133]{x}$ we need to perform

$$
V_{3}^{\mathrm{S}}(1234567890133, N)=\left(\frac{323}{4}+o(1)\right) M(N)
$$

operations.

For Householder's method the optimal value is $d=3$ and it leads to iterations

$$
a_{0}:=1 \text {, }
$$

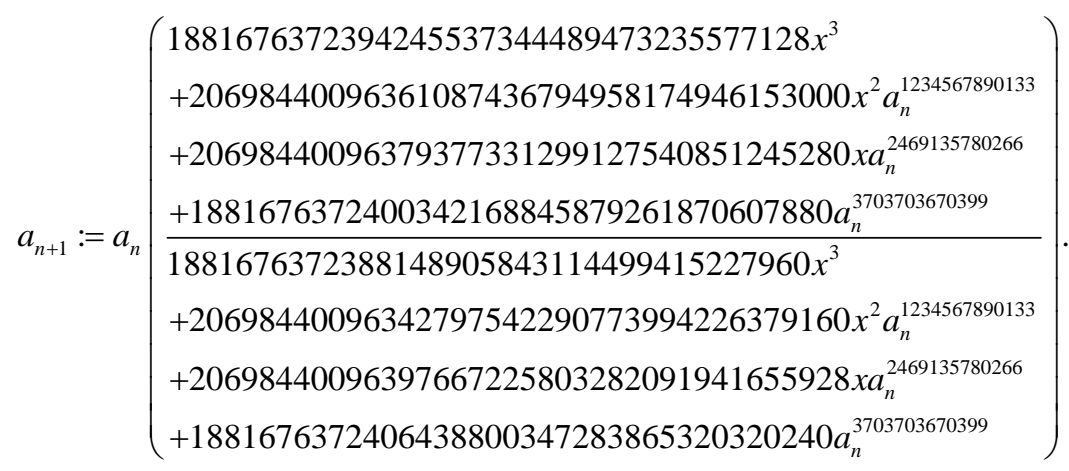


This method has order 5 and for computation of $N$ digits it needs

$$
V_{3}^{\mathrm{H}}(1234567890133, N)=\left(\frac{691}{8}+o(1)\right) M(N)
$$

operations.

Newton's method

$$
\begin{aligned}
& a_{0}:=1, \\
& a_{n+1}:=\frac{1}{1234567890133}\left(1234567890132 a_{n}+\frac{x}{a_{n}^{1234567890132}}\right)
\end{aligned}
$$

has order 2 and for computation of $N$ digits it needs (assuming that $\sigma(k-1)=59$ )

$$
V^{\mathrm{N}}(1234567890133, N)=(125+o(1)) M(N)
$$

operations.

Hence our method saves $35 \%$ of time compared to Newton's method and saves $7 \%$ of time compared to Householder's method.

\section{Proofs}

In this section we will prove that function $p$ defined by (3.1) satisfies inequalities (3.2). For the sake of brevity, will use the symbol $\mathbb{N}_{u}$ for the set $\mathbb{Z} \cap[u, \infty)$.

\subsection{Combinatorial Identities}

First we need to prove several combinatorial identities. Our notation will be changed in this subsection. Here, $n$ will be a variable used in mathematical induction, $k$ will be a summation index, and $A, B$ will be additional parameters. The change of notation is made because of easy application of the following methods based on [14]. For a function $\varphi(n, k)$ we will denote its differences by

$$
\begin{aligned}
& \Delta_{n} \varphi(n, k):=\varphi(n+1, k)-\varphi(n, k), \\
& \Delta_{k} \varphi(n, k):=\varphi(n, k+1)-\varphi(n, k) .
\end{aligned}
$$

Given a function $F(n, k)$, there is some function $G(n, k)$ satisfying some relation between $\Delta_{n} F(n, k)$ and $\Delta_{k} G(n, k)$. This new function is then used for easier evaluation of sums containing $F(n, k)$. Recall that $\frac{1}{n !}=0$ for negative integer $n$.

For $k \in \mathbb{N}_{0}$ and $n, A, B \in \mathbb{N}_{1}$ with $n \geq \max (A, B)$ put

$$
\begin{aligned}
& F_{1}(n, k, A, B):=\frac{(n-A) !(n+A-B) ! B ! A !(n-B) !(2 n-B+k) !}{(2 n-B) !(n+A) ! k !(B-k) !(n-B+k) !(A-k) !(n-A-B+k) !}, \\
& R_{1}(n, k, A, B):=\frac{k\left(2 B^{2}-6 n B-2 k B-4 B+k A-A+4 n^{2}+3 k n+5 n+3 k+1\right)}{(A+n+1)(B-2 n-2)(B-2 n-1)}, \\
& G_{1}(n, k, A, B):=F_{1}(n, k, A, B) R_{1}(n, k, A, B), \\
& S_{1}(n, A, B):=\sum_{k=0}^{n} F_{1}(n, k, A, B) .
\end{aligned}
$$

Lemma 3. For every $n, A, B \in \mathbb{N}_{1}$ satisfying $n \geq \max (A, B)$ we have

$$
S_{1}(n, A, B)=\sum_{k=0}^{n} \frac{(n-A) !(n+A-B) ! B ! A !(n-B) !(2 n-B+k) !}{(2 n-B) !(n+A) ! k !(B-k) !(n-B+k) !(A-k) !(n-A-B+k) !}=1 .
$$

Proof. From the polynomial identity 


$$
\begin{aligned}
& ((n+1-A)(n+1+A-B)(n+1-B)(2 n+1-B+k)(2 n+2-B+k) \\
- & (2 n+1-B)(2 n+2-B)(n+1+A)(n+1-B+k)(n+1-A-B+k)) \\
\times & ((k+1)(n-B+k+1)(n-A-B+k+1)(A+n+1)(B-2 n-2)(B-2 n-1)) \\
= & ((2 n+1-B)(2 n+2-B)(n+1+A)(n+1-B+k)(n+1-A-B+k)) \\
& \times\left(( 2 n - B + k + 1 ) ( B - k ) ( A - k ) ( k + 1 ) \left(2 B^{2}-6 n B-2 k B-6 B+k A\right.\right. \\
& \left.+4 n^{2}+3 k n+8 n+3 k+4\right)-k\left(2 B^{2}-6 n B-2 k B-4 B+k A-A\right. \\
& \left.\left.+4 n^{2}+3 k n+5 n+3 k+1\right)(k+1)(n-B+k+1)(n-A-B+k+1)\right)
\end{aligned}
$$

we immediately obtain

$$
\begin{aligned}
& \Delta_{n} F_{1}(n, k, A, B) \\
& =\left(\frac{(n+1-A)(n+1+A-B)(n+1-B)(2 n+1-B+k)(2 n+2-B+k)}{(2 n+1-B)(2 n+2-B)(n+1+A)(n+1-B+k)(n+1-A-B+k)}-1\right) F_{1}(n, k, A, B) \\
& =\left(\begin{array}{l}
(n+1-A)(n+1+A-B)(n+1-B)(2 n+1-B+k)(2 n+2-B+k) \\
\frac{-(2 n+1-B)(2 n+2-B)(n+1+A)(n+1-B+k)(n+1-A-B+k)}{(2 n+1-B)(2 n+2-B)(n+1+A)(n+1-B+k)(n+1-A-B+k)}
\end{array}\right) F_{1}(n, k, A, B) \\
& =\left(\begin{array}{c}
(2 n-B+k+1)(B-k)(A-k)(k+1)\left(2 B^{2}-6 n B-2 k B-6 B+k A\right. \\
\left.+4 n^{2}+3 k n+8 n+3 k+4\right)-k\left(2 B^{2}-6 n B-2 k B-4 B+k A-A\right. \\
\frac{\left.+4 n^{2}+3 k n+5 n+3 k+1\right)(k+1)(n-B+k+1)(n-A-B+k+1)}{(k+1)(n-B+k+1)(n-A-B+k+1)(A+n+1)(B-2 n-2)(B-2 n-1)}
\end{array}\right) F_{1}(n, k, A, B) \\
& =\left(\frac{(2 n-B+k+1)(B-k)(A-k)}{(k+1)(n-B+k+1)(n-A-B+k+1)} R_{1}(n, k+1, A, B)-R_{1}(n, k, A, B)\right) F_{1}(n, k, A, B) \\
& =F_{1}(n, k+1, A, B) R_{1}(n, k+1, A, B)-F_{1}(n, k, A, B) R_{1}(n, k, A, B) \\
& =\Delta_{k} G_{1}(n, k, A, B) \text {. }
\end{aligned}
$$

For fixed $k>n$ we have $\max (A, B)<k$ and hence $\frac{1}{(A-k) !}=\frac{1}{(B-k) !}=0$. Thus

$$
F_{1}(n+1, k=n+1, A, B)=0 .
$$

Similarly for $k=0$ and $k=n+1$ we have

$$
G_{1}(n, k=0, A, B)=0
$$

and

$$
G_{1}(n, k=n+1, A, B)=0 .
$$

Then (6.1), (6.2), (6.3) and (6.4) imply 


$$
\begin{aligned}
& \Delta_{n} S_{1}(n, A, B) \\
& =F_{1}(n+1, k=n+1, A, B)+\sum_{k=0}^{n} \Delta_{n} F_{1}(n, k, A, B) \\
& =F_{1}(n+1, k=n+1, A, B)+\sum_{k=0}^{n} \Delta_{k} G_{1}(n, k, A, B) \\
& =F_{1}(n+1, k=n+1, A, B)+G_{1}(n, k=n+1, A, B)-G_{1}(n, k=0, A, B) \\
& =0 .
\end{aligned}
$$

If $A=n$ and $B \leq n$ then $\frac{1}{(k-B) !}=0$ for $k<B$ and $\frac{1}{(B-k) !}=0$ for $k>B$. Hence the only nonzero summand in $S_{1}(n, A=n, B)$ is the one for $k=B$ and

$$
S_{1}(n, A=n, B)=\sum_{k=B} \frac{0 !(2 n-B) ! B ! n !(n-B) !(2 n-B+k) !}{(2 n-B) !(2 n) ! k !(B-k) !(n-B+k) !(n-k) !(k-B) !}=1 .
$$

Similarly for $A<n$ and $B=n$ we obtain

$$
S_{1}(n, A<n, B=n)=1 .
$$

From this and (6.6) we obtain that

$$
S_{1}(n=\max (A, B), A, B)=1 .
$$

Equation (6.5) implies that $S_{1}$ will not change for greater $n$.

For $n, k, A \in \mathbb{N}_{0}$ with $1 \leq A \leq n$ and $k \leq n-A$ put

$$
\begin{aligned}
& F_{2}(n, k, A):=\frac{(-1)^{k+n-A}(n+k) !(n+1-A) !(n+1) !}{k !(A+k) !(n+1-A-k) !(2 n+1-A) !}, \\
& T_{2}(n, k, A):=\frac{k(A+k)}{(n+1)(A-n-1)}, \\
& S_{2}(n, A):=\sum_{k=0}^{n-A} F_{2}(n, k, A) .
\end{aligned}
$$

Lemma 4. For every $n, A \in \mathbb{N}_{1}$ with $A \leq n$

$$
S_{2}(n, A)=\sum_{k=0}^{n-A} \frac{(-1)^{k+n-A}(n+k) !(n+1-A) !(n+1) !}{k !(A+k) !(n+1-A-k) !(2 n+1-A) !}=1 .
$$

Proof. From the polynomial identity

we obtain for $k \leq n-A$ that

$$
(n+k+1)(n+1-A-k)+k(A+k)=-(n+1)(A-n-1) .
$$

$$
\Delta_{k}\left(F_{2}(n, k, A) T_{2}(n, k, A)\right)=-\frac{(n+k+1)(n+1-A-k)+k(A+k)}{(n+1)(A-n-1)} F_{2}(n, k, A)=F_{2}(n, k, A) .
$$

This and the fact that $T_{2}(n, k=0, A)=0$ imply

$$
\begin{aligned}
S_{2}(n, A) & =\sum_{k=0}^{n-A} F_{2}(n, k, A)=\sum_{k=0}^{n-A} \Delta_{k}\left(F_{2}(n, k, A) T_{2}(n, k, A)\right) \\
& =F_{2}(n, k=n-A+1, A) T_{2}(n, k=n-A+1, A)-F_{2}(n, k=0, A) T_{2}(n, k=0, A) \\
& =\frac{(-1)^{2 n-2 A+1}(2 n-A+1) !(n+1-A) !(n+1) !(n-A+1)(n+1)}{(n-A+1) !(n+1) ! 0 !(2 n+1-A) !(n+1)(A-n-1)}=1 .
\end{aligned}
$$


For $n, k, A, B \in \mathbb{N}_{0}$ with $1 \leq A \leq B<n$ and $k \leq n-A$ put

$$
\begin{aligned}
& F_{3}(n, k, A, B):=\frac{(-1)^{k}(B+k) !(n-k-1) !}{(B-k) ! k !(A+k) !(n-A-k) !}, \\
& G_{3}(n, k, A, B):=\frac{(-1)^{k+1}(B+k) !(n-k) !}{(B-k) !(k-1) !(A+k-1) !(n-A-k+1) !}, \\
& S_{3}(n, A, B):=\sum_{k=0}^{n-A} F_{3}(n, k, A, B) .
\end{aligned}
$$

Lemma 5. For every $n, A, B \in \mathbb{N}_{1}$ with $A \leq B<n$

$$
S_{3}(n, A, B)=\sum_{k=0}^{n-A} \frac{(-1)^{k}(B+k) !(n-k-1) !}{(B-k) ! k !(A+k) !(n-A-k) !}=0 .
$$

Proof. From the polynomial identity

$$
\begin{aligned}
& (B-n)(B+n+1)(n-A-k+1)-(n+1)(A-n-1)(n-k) \\
& =(B+k+1)(B-k)(n-A-k+1)+(n-k) k(A+k)
\end{aligned}
$$

we obtain for $A \leq B<n$ and $k \leq n-A$ that

$$
\begin{aligned}
\Delta_{k} G_{3}(n, k, A, B) & =\frac{(-1)^{k}(B+k+1) !(n-k-1) !}{(B-k-1) ! k !(A+k) !(n-A-k) !}-\frac{(-1)^{k+1}(B+k) !(n-k) !}{(B-k) !(k-1) !(A+k-1) !(n-A-k+1) !} \\
& =\left((B+k+1)(B-k)+\frac{(n-k) k(A+k)}{n-A-k+1}\right) F_{3}(n, k, A, B) \\
& =\left((B-n)(B+n+1)-\frac{(n+1)(A-n-1)(n-k)}{n-A-k+1}\right) F_{3}(n, k, A, B) \\
& =(B-n)(B+n+1) F_{3}(n, k, A, B)-(n+1)(A-n-1) F_{3}(n+1, k, A, B) .
\end{aligned}
$$

For $k=0$ we have $G_{3}(n, k=0, A, B)=0$. This and (6.7) imply

$$
\begin{aligned}
& (B-n)(B+n+1) S_{3}(n, A, B)-(n+1)(A-n-1) S_{3}(n+1, A, B) \\
= & \sum_{k=0}^{n-A}\left((B-n)(B+n+1) F_{3}(n, k, A, B)-(n+1)(A-n-1) F_{3}(n+1, k, A, B)\right) \\
& -(n+1)(A-n-1) F_{3}(n+1, k=n-A+1, A, B) \\
= & \sum_{k=0}^{n-A} \Delta_{k} G_{3}(n, k, A, B)-(n+1)(A-n-1) F_{3}(n+1, k=n-A+1, A, B) \\
= & G_{3}(n, k=n-A+1, A, B)-G_{3}(n, k=0, A, B)-(n+1)(A-n-1) F_{3}(n+1, k=n-A+1, A, B) \\
= & \frac{(-1)^{n-A}(n-A+B+1) !(A-1) !}{(A+B-n-1) !(n-1) ! n ! 0 !}-\frac{(-1)^{n-A+1}(n+1)(A-n-1)(n-A+B+1) !(A-1) !}{(A+B-n-1) !(n-A+1) !(n+1) ! 1 !}=0 .
\end{aligned}
$$

Lemma 4 for $n=B$ implies

$$
\sum_{k=0}^{B-A} \frac{(-1)^{k+B-A}(B+k) !(B+1-A) !(B+1) !}{k !(A+k) !(B+1-A-k) !(2 B+1-A) !}=1,
$$

hence

$$
\sum_{k=0}^{B-A} \frac{(-1)^{k}(B+k) !}{k !(A+k) !(B+1-A-k) !}=\frac{(-1)^{B-A}(2 B+1-A) !}{(B+1-A) !(B+1) !}
$$

and 


$$
S_{3}(n=B+1, A, B)=\sum_{k=0}^{B-A+1} \frac{(-1)^{k}(B+k) !}{k !(A+k) !(B+1-A-k) !}=0 .
$$

For $n \geq B+1$ we have $A-n-1>0$ and (6.8) yields

$$
S_{3}(n+1, A, B)=\frac{(B-n)(B+n+1)}{(n+1)(A-n-1)} S_{3}(n, A, B),
$$

This with (6.9) implies the result for every $n>B$.

For $n, k, A \in \mathbb{N}_{0}$ with $A \leq n$ put

$$
\begin{aligned}
& F_{4}(n, k, A):=\frac{(2 n-k) ! k !(n-A) !(A+n+1) !}{(k-A) !(n-k) ! n !(2 n+1) ! A !}, \\
& G_{4}(n, k, A):=-\frac{(2 n-k+1) ! k !(n-A) !(A+n+1) !}{(k-A-1) !(n-k+1) !(n+1) !(2 n+3) ! A !}, \\
& S_{4}(n, A):=\sum_{k=A}^{n} F_{4}(n, k, A) .
\end{aligned}
$$

Lemma 6. For every $A, n \in \mathbb{N}_{0}$ with $A \leq n$

$$
S_{4}(n, A)=\sum_{k=A}^{n} \frac{(2 n-k) ! k !(n-A) !(A+n+1) !}{(k-A) !(n-k) ! n !(2 n+1) ! A !}=1 .
$$

Proof. From the polynomial identity

$$
\begin{aligned}
& (2 n-k+1)(2 n-k+2)(n-A+1)(A+n+2)-(n+1-k)(n+1)(2 n+2)(2 n+3) \\
= & (2 n-k+1)(2 n A-k A+2 A+k n+n+k+1)(k-A) \\
& -(k+1)(2 n A-k A+A+k n+2 n+k+2)(n-k+1)
\end{aligned}
$$

we obtain for $0 \leq A \leq n$ and $k \in \mathbb{N}_{0}$ that

$$
\begin{aligned}
& \Delta_{n} F_{4}(n, k, A) \\
= & \frac{(2 n-k+1)(2 n-k+2)(n-A+1)(A+n+2)-(n+1-k)(n+1)(2 n+2)(2 n+3)}{(n+1-k)(n+1)(2 n+2)(2 n+3)} F_{4}(n, k, A) \\
= & \left(\begin{array}{l}
-(k+1)(2 n A-k A+A+k n+2 n+k+2)(n-k+1) \\
+(2 n-k+1)(2 n A-k A+2 A+k n+n+k+1)(k-A) \\
(n-k+1)(n+1)(2 n+2)(2 n+3)
\end{array}\right) F_{4}(n, k, A) \\
= & \left(-\frac{(k+1)(2 n A-k A+A+k n+2 n+k+2)}{(n+1)(2 n+2)(2 n+3)}+\frac{(2 n-k+1)(2 n A-k A+2 A+k n+n+k+1)(k-A)}{(n-k+1)(n+1)(2 n+2)(2 n+3)}\right) F_{4}(n, k, A) \\
= & -\frac{(2 n-k) !(k+1) !(n-A) !(A+n+1) !(2 n A-k A+A+k n+2 n+k+2)}{(k-A) !(n-k) !(n+1) !(2 n+3) ! A !} \\
& +\frac{(2 n-k+1) ! k !(n-A) !(A+n+1) !(2 n A-k A+2 A+k n+n+k+1)}{(k-A-1) !(n-k+1) !(n+1) !(2 n+3) ! A !} \\
= & \Delta_{k} G_{4}(n, k, A) .
\end{aligned}
$$

For $k=A$ we have $G_{4}(n, k=A, A)=0$. From this, (6.10) and the polynomial identity 


$$
2 n A-(n+1) A+2 A+(n+1) n+2 n+2=(n+1)(A+n+2)
$$

we obtain

$$
\begin{aligned}
\Delta_{n} S_{4}(n, A)= & F_{4}(n+1, k=n+1, A)+\sum_{k=A}^{n} \Delta_{n} F_{4}(n, k, A) \\
= & F_{4}(n+1, k=n+1, A)+\sum_{k=A}^{n} \Delta_{k} G_{4}(n, k, A) \\
= & F_{4}(n+1, k=n+1, A)+G_{4}(n, k+1, A)-G_{4}(n, k=A, A) \\
= & \frac{(n+1) !(n+1) !(n+1-A) !(A+n+2) !}{(n+1-A) ! 0 !(n+1) !(2 n+3) ! A !} \\
& -\frac{n !(n+1) !(n-A) !(A+n+1) !(2 n A-(n+1) A+2 A+(n+1) n+2 n+2)}{(n-A) ! 0 !(n+1) !(2 n+3) ! A !} \\
= & \frac{(n+1) !(A+n+2) !}{(2 n+3) ! A !}-\frac{n !(A+n+1) !(n+1)(A+n+2)}{(2 n+3) ! A !} \\
= & 0 .
\end{aligned}
$$

For $n=A$ the sum $S_{4}(n, A)$ contains only one nonzero summand for $k=A$ and we have $S_{4}(n=A, A)=1$. Equation (6.11) implies that $S_{4}$ will not change for greater $n$.

For $n, k, A, B \in \mathbb{N}_{0}$ with $A \leq B<n$ put

$$
\begin{aligned}
& F_{5}(n, k, A, B):=\frac{(2 B-k) !(n-B+k-1) !(B-A) !(n+A) !}{(k-A) !(B-k) ! B !(n+B) !(n-B+A-1) !} \\
& G_{5}(n, k, A, B):=-\frac{(2 B-k+1) !(n-B+k-1) !(B-A) !(n+A) !}{(k-A-1) !(B-k) ! B !(n+B+1) !(n-B+A) !}, \\
& S_{5}(n, A, B):=\sum_{k=A}^{B} F_{5}(n, k, A, B) .
\end{aligned}
$$

Lemma 7. For every $A, B, n \in \mathbb{N}_{0}$ with $A \leq B<n$

$$
S_{5}(n, A, B)=\sum_{k=A}^{B} \frac{(2 B-k) !(n-B+k-1) !(B-A) !(n+A) !}{(k-A) !(B-k) ! B !(n+B) !(n-B+A-1) !}=1 .
$$

Proof. From the polynomial identity

$$
(n-B+k)(n+A+1)-(n+B+1)(n-B+A)=(2 B-k+1)(k-A)-(n-B+k)(B-k)
$$

we obtain for $0 \leq A \leq B<n$ and $k \in \mathbb{N}_{0}$

$$
\begin{aligned}
& \Delta_{n} F_{5}(n, k, A, B) \\
= & \frac{(n-B+k)(n+A+1)-(n+B+1)(n-B+A)}{(n+B+1)(n-B+A)} F_{5}(n, k, A, B) \\
= & \left(-\frac{(n-B+k)(B-k)}{(n+B+1)(n-B+A)}+\frac{(2 B-k+1)(k-A)}{(n+B+1)(n-B+A)}\right) F_{5}(n, k, A, B) \\
= & -\frac{(2 B-k) !(n-B+k) !(B-A) !(n+A) !}{(k-A) !(B-k-1) ! B !(n+B+1) !(n-B+A) !} \\
& +\frac{(2 B-k+1) !(n-B+k-1) !(B-A) !(n+A) !}{(k-A-1) !(B-k) ! B !(n+B+1) !(n-B+A) !} \\
= & \Delta_{k} G_{5}(n, k, A, B) .
\end{aligned}
$$


For $k=A$ and $k=B+1$ we have

$$
G_{5}(n, k=A, A, B)=0
$$

and

$$
G_{5}(n, k=B+1, A, B)=0 .
$$

From this and (6.12) we obtain

$$
\begin{aligned}
& \Delta_{n} S_{5}(n, A, B) \\
= & \sum_{k=A}^{B} \Delta_{n} F_{5}(n, A, B)=\sum_{k=A}^{B} \Delta_{n} G_{5}(n, A, B) \\
= & G_{5}(n, k=B+1, A, B)-G_{5}(n, k=A, A, B)=0 .
\end{aligned}
$$

Lemma 6 for $n=B$ yields

$$
\begin{aligned}
& S_{5}(n=B+1, A, B) \\
& =\sum_{k=A}^{B} \frac{(2 B-k) ! k !(B-A) !(A+B+1) !}{(k-A) !(B-k) ! B !(2 B+1) ! A !}=S_{4}(n=B, A)=1 .
\end{aligned}
$$

Equation (6.13) implies that $S_{5}(n, A, B)$ has the same value for greater $n$.

For $n, B \in \mathbb{N}_{1}$ and $k \in \mathbb{N}_{0}$ put

$$
\begin{aligned}
F_{6}(n, k, B):= & \frac{(-1)^{k}(n+k) !(2 n+B) !^{2}(B-1) !(2 n+B-k-1) ! n !}{(n-k) !^{2} k !(n+B+k) !(n+B-1) !^{2}(3 n+B) !}, \\
T_{6}(n, k, B):= & 3 n B^{4}-k B^{4}+3 B^{4}+24 n^{2} B^{3}-9 k n B^{3}+39 n B^{3}-k^{2} B^{3}-6 k B^{3} \\
& +15 B^{3}+69 n^{3} B^{2}-26 k n^{2} B^{2}+151 n^{2} B^{2}-7 k^{2} n B^{2}-30 k n B^{2} \\
& +104 n B^{2}-5 k^{2} B^{2}-7 k B^{2}+22 B^{2}+84 n^{4} B-28 k n^{3} B+225 n^{3} B \\
& -16 k^{2} n^{2} B-38 k n^{2} B+211 n^{2} B-23 k^{2} n B-9 k n B+80 n B-8 k^{2} B \\
& +2 k B+10 B+37 n^{5}-10 k n^{4}+116 n^{4}-11 k^{2} n^{3}-14 k n^{3}+136 n^{3} \\
& -22 k^{2} n^{2}-2 k n^{2}+74 n^{2}-13 k^{2} n+2 k n+19 n-2 k^{2}+2, \\
G_{6}(n, k, B):= & \frac{(-1)^{k+1}(n+k) !(2 n+B) !^{2}(B-1) !(2 n+B-k) ! n ! T_{6}(n, k, B)}{(n-k+1) !^{2}(k-1) !(n+B+k) !(n+B) !^{2}(3 n+B+3) !}, \\
S_{6}(n, B):=\sum_{k=0}^{n} & F_{6}(n, k, B) .
\end{aligned}
$$

Lemma 8. For every $n, B \in \mathbb{N}_{1}$

$$
S_{6}(n, B)=\sum_{k=0}^{n} \frac{(-1)^{k}(n+k) !(2 n+B) !^{2}(B-1) !(2 n+B-k-1) ! n !}{(n-k) !^{2} k !(n+B+k) !(n+B-1) !^{2}(3 n+B) !}=1 .
$$

Proof. From the polynomial identity

$$
\begin{aligned}
& (n+k+1)(2 n+B+1)^{2}(2 n+B+2)^{2}(2 n+B-k)(2 n+B-k+1)(n+1) \\
& -(n-k+1)^{2}(n+B+k+1)(n+B)^{2}(3 n+B+1)(3 n+B+2)(3 n+B+3) \\
& =(n+k+1) T_{6}(n, k+1, B)(n-k+1)^{2}+(2 n+B-k) T_{6}(n, k, B) k(n+B+k+1)
\end{aligned}
$$

we obtain 


$$
\begin{aligned}
& \Delta_{n} F_{6}(n, k, B) \\
& =\left(\begin{array}{l}
(n+k+1)(2 n+B+1)^{2}(2 n+B+2)^{2}(2 n+B-k)(2 n+B-k+1)(n+1) \\
\frac{-(n-k+1)^{2}(n+B+k+1)(n+B)^{2}(3 n+B+1)(3 n+B+2)(3 n+B+3)}{(n-k+1)^{2}(n+B+k+1)(n+B)^{2}(3 n+B+1)(3 n+B+2)(3 n+B+3)}
\end{array}\right) F_{6}(n, k, B) \\
& =\frac{(n+k+1) T_{6}(n, k+1, B)(n-k+1)^{2}+(2 n+B-k) T_{6}(n, k, B) k(n+B+k+1)}{(n+B+k+1)(n-k+1)^{2}(n+B)^{2}(3 n+B+1)(3 n+B+2)(3 n+B+3)} F_{6}(n, k, B) \\
& =\left(\frac{(n+k+1) T_{6}(n, k+1, B)}{(n+B+k+1)(n+B)^{2}(3 n+B+1)(3 n+B+2)(3 n+B+3)}\right. \\
& \left.+\frac{(2 n+B-k) T_{6}(n, k, B) k}{(n-k+1)^{2}(n+B)^{2}(3 n+B+1) \times(3 n+B+2)(3 n+B+3)}\right) F_{6}(n, k, B) \\
& =\frac{(-1)^{k+2}(n+k+1) !(2 n+B) !^{2}(B-1) !(2 n+B-k-1) ! n ! T_{6}(n, k+1, B)}{(n-k) !^{2} k !^{2}(n+B+k+1) !(n+B) !^{2}(3 n+B+3) !} \\
& -\frac{(-1)^{k+1}(n+k) !(2 n+B) !^{2}(B-1) !(2 n+B-k) ! n ! T_{6}(n, k, B)}{(n-k+1) !^{2}(k-1) !(n+B+k) !(n+B) !^{2}(3 n+B+3) !} \\
& =\Delta_{k} G_{6}(n, k, B) \text {. }
\end{aligned}
$$

For $k=0$ we have $G_{6}(n, k=0, B)=0$. From this, (6.14) and the polynomial identity

$$
T_{6}(n, k=n+1, B)=(2 n+2)(2 n+B+1)^{2}(2 n+B+2)(n+B)
$$

we obtain

$$
\begin{aligned}
& \Delta_{n} S_{6}(n, B)=F_{6}(n+1, k=n+1, B)+\sum_{k=0}^{n} \Delta_{n} F_{6}(n, k, B) \\
&=F_{6}(n+1, k=n+1, B)+\sum_{k=0}^{n} \Delta_{k} G_{6}(n, k, B) \\
&=F_{6}(n+1, k=n+1, B)+G_{6}(n, k=n+1, B)-G_{6}(n, k=0, B) \\
&=\frac{(-1)^{n+1}(2 n+2) !(2 n+B+2) !^{2}(B-1) !(n+B) !(n+1) !}{0 !^{2}(n+1) !(2 n+B+2) !(n+B) !^{2}(3 n+B+3) !} \\
&+\frac{(-1)^{n+2}(2 n+1) !(2 n+B) !^{2}(B-1) !(n+B-1) ! n ! T_{6}(n, k=n+1, B)}{0 !^{2} n !(2 n+B+1) !(n+B) !^{2}(3 n+B+3) !} \\
&=\frac{(-1)^{n+1}(2 n+1) !(2 n+B) !^{2}(B-1) !(n+B-1) !}{(2 n+B+1) !(n+B) !^{2}(3 n+B+3) !} \\
& \quad \times\left((2 n+2)(2 n+B+1)^{2}(2 n+B+2)(n+B)-T_{6}(n, k=n+1, B)\right) \\
&= 0 .
\end{aligned}
$$

For $n=1$ we have

$$
S_{6}(n=1, B)=\frac{(B-1) !(B+2) !^{2}}{B !^{2}(B+3) !}-\frac{2(B-1) !(B+2) !}{B !(B+3) !}=\frac{(B+1)(B+2)-2}{B(B+3)}=1 .
$$

Equation (6.15) implies that $S_{6}(n, B)$ has the same value for greater $n$. 
For $n, A, B \in \mathbb{N}_{1}$ and $k \in \mathbb{N}_{0}$ with $n \geq 2 A+B$ put

$$
\begin{aligned}
& F_{7}(n, k, A, B):=\frac{(-1)^{k}(A+k) !(2 A+B) !(B-1) !(n-k-1) !(n-A-B) ! n !}{(A-k) ! k !(A+B+k) !(A+B-1) !(n-A-1) !(n-A-B-k) !(n+A) !}, \\
& G_{7}(n, k, A, B):=\frac{(-1)^{k+1}(A+k) !(2 A+B) !(B-1) !(n-k) !(n-A-B) ! n !}{(A-k) !(k-1) !(A+B+k-1) !(A+B+1) !(n-A) !(n-A-B-k+1) !(n+A+1) !}, \\
& S_{7}(n, A, B):=\sum_{k=0}^{A} F_{7}(n, k, A, B) .
\end{aligned}
$$

Lemma 9. For every $A, B, n \in \mathbb{N}_{1}$ with $n \geq 2 A+B$

$$
S_{7}(n, A, B)=\sum_{k=0}^{A} \frac{(-1)^{k}(A+k) !(2 A+B) !(B-1) !(n-k-1) !(n-A-B) ! n !}{(A-k) ! k !(A+B+k) !(A+B-1) !(n-A-1) !(n-A-B-k) !(n+A) !}=1 .
$$

Proof. From the polynomial identity

$$
\begin{aligned}
& (n-k)(n-A-B+1)(n+1)-(n-A)(n-A-B-k+1)(n+A+1) \\
& =(A+k+1)(A-k)(n-A-B-k+1)+(n-k) k(A+B+k)
\end{aligned}
$$

we obtain for $n, A, B \in \mathbb{N}_{1}$ and $k \in \mathbb{N}_{0}$ with $n \geq 2 A+B$

$$
\begin{aligned}
& \Delta_{n} F_{7}(n, k, A, B) \\
= & \frac{(n-k)(n-A-B+1)(n+1)-(n-A)(n-A-B-k+1)(n+A+1)}{(n-A)(n-A-B-k+1)(n+A+1)} F_{7}(n, k, A, B) \\
= & \frac{(A+k+1)(A-k)(n-A-B-k+1)+(n-k) k(A+B+k)}{(n-A)(n-A-B-k+1)(n+A+1)} F_{7}(n, k, A, B) \\
= & \left(\frac{(A+k+1)(A-k)}{(n-A)(n+A+1)}+\frac{(n-k) k(A+B+k)}{(n-A)(n-A-B-k+1)(n+A+1)}\right) F_{7}(n, k, A, B) \\
= & \frac{(-1)^{k+2}(A+k+1) !(2 A+B) !(B-1) !(n-k-1) !(n-A-B) ! n !}{(A-k-1) ! k !(A+B+k) !(A+B+1) !(n-A) !(n-A-B-k) !(n+A+1) !} \\
& -\frac{(-1)}{(A-k) !(k-1) !(A+B+k-1) !(A+B+1) !(n-A) !(n-A-B-k+1) !(n+A+1) !} \\
= & \Delta_{k} G_{7}(n, k, A, B) .
\end{aligned}
$$

For $k=0$ and $k=A+1$ we have

$$
G_{7}(n, k=0, A, B)=0
$$

and

$$
G_{7}(n, k=A+1, A, B)=0 .
$$

From this and (6.16) we obtain

$$
\begin{aligned}
\Delta_{n} S_{7}(n, A, B) & =\sum_{k=0}^{A} \Delta_{n} F_{7}(n, k, A, B)=\sum_{k=0}^{A} \Delta_{k} G_{7}(n, k, A, B) \\
& =G_{7}(n, k=A+1, A, B)-G_{7}(n, k=0, A, B)=0 .
\end{aligned}
$$

For $n=2 A+B$ Lemma 8 implies 


$$
\begin{aligned}
& S_{7}(n=2 A+B, A, B) \\
& =\sum_{k=0}^{A} \frac{(-1)^{k}(A+k) !(2 A+B) !(B-1) !(2 A+B-k-1) ! A !(2 A+B) !}{(A-k) ! k !(A+B+k) !(A+B-1) !(A+B-1) !(A-k) !(3 A+B) !} \\
& =S_{6}(n=A, B)=1 .
\end{aligned}
$$

Equation (6.17) implies that $S_{7}(n, B)$ has the same value for greater $n$.

\subsection{Formulas}

Now the symbol $k$ again has its original meaning.

For $k \in \mathbb{N}_{2}$ and $i \in \mathbb{N}_{1}$ put

$$
g_{0, k}:=1 \quad \text { and } \quad g_{i, k}:=\frac{(-1)^{i-1}}{k^{i} i !} \prod_{j=1}^{i-1}(j k-1)
$$

The numbers $g_{i, k}$ are the coefficients of Taylor's polynomial of the function $\sqrt[k]{t}$.

Lemma 10. For $k \in \mathbb{N}_{2}$ and $t \in(0,2)$

$$
\sqrt[k]{t}=\sum_{i=0}^{\infty} g_{i, k}(t-1)^{i}
$$

Proof. See for instance Theorem 159 in [15].

Now we prove a technical lemma that we need later.

Lemma 11. For $k \in \mathbb{R}, s \in \mathbb{N}_{1}$ and $j=1, \cdots, s$

$$
\begin{aligned}
& \frac{(2 s-j) !}{j !(s-j) !} \prod_{h=s-j+1}^{s}(h k+1)=\frac{(2 s-j) !}{j !(s-j) !} \prod_{h=s-j+1}^{s}(h k-1) \\
& -\sum_{i=1}^{j}\left(\frac{(-1)^{i}}{i !} \frac{(2 s-j+i) !}{(j-i) !(s-j+i) !} \prod_{h=1}^{i-1}(h k-1) \prod_{h=s-j+i+1}^{s}(h k-1)\right) .
\end{aligned}
$$

Proof. On both sides of (6.18) there are polynomials of degree $j$ in variable $k$. Therefore it suffices to prove the equality for $k \rightarrow \infty$ and for $j$ values $k=\frac{1}{m}$ with $m=s-j+1, \cdots, s$.

For $k \rightarrow \infty$ we immediately have

$$
\text { LHS } \sim \frac{(2 s-j) !}{j !(s-j) !} k^{j} \prod_{h=s-j+1}^{s} h \sim \text { RHS. }
$$

For $k=\frac{1}{m}$ with $m=s-j+1, \cdots, s$ we obtain on the left-hand side of (6.18)

$$
\text { LHS }=\frac{(2 s-j) !}{j !(s-j) !} \prod_{h=s-j+1}^{s}\left(\frac{h}{m}+1\right)=\frac{(2 s-j) !}{j !(s-j) !} \frac{1}{m^{j}} \frac{(m+s) !}{(m+s-j) !} .
$$

For such numbers $m$ we have

$$
\prod_{h=s-j+1}^{s}\left(\frac{h}{m}-1\right)=0 .
$$

Therefore we obtain on the right-hand side of (6.18)

$$
\begin{aligned}
\text { RHS } & =\frac{(2 s-j) !}{j !(s-j) !} \prod_{h=s-j+1}^{s}\left(\frac{h}{m}-1\right)-\sum_{i=1}^{j} \frac{(-1)^{i}}{i !}\left(\frac{(2 s-j+i) !}{(j-i) !(s-j+i) !} \prod_{h=1}^{i-1}\left(\frac{h}{m}-1\right) \prod_{h=s-j+i+1}^{s}\left(\frac{h}{m}-1\right)\right) \\
& =\sum_{i=1}^{j}\left(\frac{1}{i !} \frac{(2 s-j+i) !}{(j-i) !(s-j+i) !} \frac{1}{m^{j-1}} \prod_{h=1}^{i-1}(m-h) \prod_{h=s-j+i+1}^{s}(h-m)\right) .
\end{aligned}
$$


The first product on the last line is equal to zero for $i \geq m+1$, the second product on the last line is equal to zero for $i \leq m+j-s-1$. For other values of $i$ both products contain only positive terms. Hence

$$
\begin{aligned}
\text { LHS } & \left.=\sum_{i=m-s+j}^{\min (j, m)} \frac{1}{i !(j-i) !(s-j+i) !} \frac{1}{m^{j-1}} \prod_{h=1}^{i-1}(m-h) \prod_{h=s-j+i+1}^{s}(h-m)\right) \\
& =\sum_{i=m-s+j}^{\min (j, m)} \frac{(2 s-j+i) ! m !(s-m) !}{i !(j-i) !(s-j+i) ! m^{j}(m-i) !(s-m-j+i) !} .
\end{aligned}
$$

This implies that

$$
\frac{\mathrm{RHS}}{\mathrm{LHS}}=\sum_{i=m-s+j}^{\min (j, m)} \frac{(2 s-j+i) ! m !(s-m) ! j !(s-j) !(m+s-j) !}{i !(j-i) !(s-j+i) !(m-i) !(s-m-j+i) !(2 s-j) !(m+s) !} .
$$

For $i<m-s+j$ we have $\frac{1}{(s-m-j+i) !}=0$, for $i>j$ we have $\frac{1}{(j-i) !}=0$ and for $i>m$ we have $\frac{1}{(s-i) !}=0$. Then Lemma 3 implies

$$
\begin{aligned}
1 & =S_{1}(n=s, A=m, B=j) \\
& =\sum_{i=0}^{s} \frac{(2 s-j+i) ! m !(s-m) ! j !(s-j) !(m+s-j) !}{i !(j-i) !(s-j+i) !(m-i) !(s-m-j+i) !(2 s-j) !(m+s) !} \\
& =\sum_{i=m-s+j}^{\min (j, m)} \frac{(2 s-j+i) ! m !(s-m) ! j !(s-j) !(m+s-j) !}{i !(j-i) !(s-j+i) !(m-i) !(s-m-j+i) !(2 s-j) !(m+s) !} \\
& =\frac{\text { RHS }}{\text { LHS }}
\end{aligned}
$$

Hence equality (6.18) follows for $k=\frac{1}{m}$ with $m=s-j+1, \cdots, s$.

For $k \in \mathbb{N}_{2}, s \in \mathbb{N}_{1}$ and $j=0, \cdots, s$ put

$$
\begin{aligned}
& c_{j, k, s}:=\frac{(2 s-j) !}{j !(s-j) !} k^{s-j} \prod_{h=s-j+1}^{s}(h k+1), \\
& d_{j, k, s}:=\frac{(2 s-j) !}{j !(s-j) !} k^{s-j} \prod_{h=s-j+1}^{s}(h k-1) .
\end{aligned}
$$

For $k \in \mathbb{N}_{2}$ and $s \in \mathbb{N}_{1}$ put

$$
p_{k, s}(t):=\frac{\sum_{j=0}^{s} c_{j, k, s}(t-1)^{j}}{\sum_{j=0}^{s} d_{j, k, s}(t-1)^{j}} .
$$

We will prove that (6.19) is Padé approximation of $\sqrt[k]{t}$.

Lemma 12. For $k \in \mathbb{N}_{2}, \quad s \in \mathbb{N}_{1}$ the numbers $c_{j, k, s}$ and $d_{j, k, s}$ satisfy the system of equations

$$
\sum_{i=0}^{j} d_{j-i, k, s} g_{i, k}=c_{j, k, s}, \quad j=0, \cdots, s .
$$

Proof. Lemma 11 implies 


$$
\begin{aligned}
c_{j, k, s}= & \frac{(2 s-j) !}{j !(s-j) !} k^{s-j} \prod_{h=s-j+1}^{s}(h k+1) \\
= & \frac{(2 s-j) !}{j !(s-j) !} k^{s-j} \prod_{h=s-j+1}^{s}(h k-1)-\sum_{i=1}^{j}\left(\frac{(-1)^{i}}{i !} \frac{(2 s-j+i) !}{(j-i) !(s-j+i) !} k^{s-j}\right. \\
& \left.\times \prod_{h=1}^{i-1}(h k-1) \prod_{h=s-j+i+1}^{s}(h k-1)\right) \\
= & \frac{(2 s-j) !}{j !(s-j) !} k^{s-j} \prod_{h=s-j+1}^{s}(h k-1)+\sum_{i=1}^{j}\left(\frac{(2 s-j+i) !}{(j-i) !(s-j+i) !} k^{s-j+i}\right. \\
& \left.\times \prod_{h=s-j+i+1}^{s}(h k-1) \frac{(-1)^{i-1}}{k^{i} i !} \prod_{h=1}^{i-1}(h k-1)\right) \\
= & d_{j, k, s} g_{0, k}+\sum_{i=1}^{j} d_{j-i, k, s} g_{i, k}
\end{aligned}
$$

Lemma 13. For $k \in \mathbb{N}_{2}, \quad s \in \mathbb{N}_{1}$ the numbers $c_{j, k, s}$ and $d_{j, k, s}$ satisfy the system of equations

$$
\sum_{i=0}^{s} d_{s-i, k, s} g_{j+i, k}=0, \quad j=1, \cdots, s .
$$

Proof. For $j=1, \cdots, s$ we obtain on the left-hand side

$$
\text { LHS }=\sum_{i=0}^{s} d_{s-i, k, s} g_{j+i}=\sum_{i=0}^{s}\left(\frac{(s+i) !}{(s-i) ! i !} k^{i} \frac{(-1)^{i+j-1}}{k^{i+j}(i+j) !} \prod_{h=i+1}^{s}(h k-1) \prod_{h=1}^{i+j-1}(h k-1)\right) .
$$

This expression, multiplied by $k^{j}$, is a polynomial of degree $s+j-1$ in variable $k$. Therefore it suffices to prove the equality for $s+j$ values $k=\frac{1}{m}$ with $m=1, \cdots, s+j$. For $m \leq s$ we have $\prod_{h=1}^{s}\left(h-\frac{1}{k}\right)=0$ and hence the whole expression is equal to zero. For $m \geq s+1$ we obtain

$$
\text { LHS }=\left(k^{s-1} \prod_{h=1}^{s}(h-m)\right) \sum_{i=0}^{s} \frac{(-1)^{i+j-1}(s+i) !}{(s-i) ! i !(i+j) !} \prod_{h=i+1}^{i+j-1}(h-m) .
$$

The second product is equal to zero for $i>m-j$, therefore the summations ends for $i=m-j$. Then Lemma 5 implies

$$
\begin{aligned}
\text { LHS } & =\left(k^{s-1} \prod_{h=1}^{s}(h-m)\right) \sum_{i=0}^{m-j} \frac{(-1)^{i}(s+i) !}{(s-i) ! i !(i+j) !} \prod_{h=i+1}^{i+j-1}(m-h) \\
& =\sum_{i=0}^{m-j} \frac{(-1)^{i}(s+i) !(m-i-1) !}{(s-i) ! i !(i+j) !(m-i-j) !}=S_{3}(n=m, A=j, B=s)=0 .
\end{aligned}
$$

Lemma 14. Function $p_{k, s}(t)$ is the Padé approximation of the function $\sqrt[k]{t}$ of order $[s, s]$ around $t=1$. Proof. For $t \rightarrow 1$ Lemma 10, Lemma 12 and Lemma 13 imply

$$
\begin{aligned}
& \left(\sum_{i=0}^{s} d_{i, k, s}(t-1)^{i}\right) \sqrt[k]{t}=\left(\sum_{i=0}^{s} d_{i, k, s}(t-1)^{i}\right)\left(\sum_{i=0}^{\infty} g_{i, k}(t-1)^{i}\right) \\
& =\sum_{j=0}^{s}(t-1)^{j} \sum_{i=0}^{j} d_{j-i, k, s} g_{i, k}+\sum_{j=1}^{s}(t-1)^{s+j} \sum_{i=0}^{s} d_{s-i, k, s} g_{j+i, k}+O(t-1)^{2 s+1} \\
& =\sum_{j=0}^{s} c_{j, k, s}(t-1)^{j}+O(t-1)^{2 s+1} .
\end{aligned}
$$

The result follows. 
Now we find the coefficients $e_{i, k, s}$ and $f_{i, k, s}$ of the Padé approximation

$$
p_{k, s}(t)=: \frac{\sum_{i=0}^{s} e_{i, k, s} t^{i}}{\sum_{i=0}^{s} f_{i, k, s} t^{i}} .
$$

Lemma 15. For every $s \in \mathbb{N}_{1}, i=0, \cdots, s$ and $k \in \mathbb{R}$

$$
\begin{aligned}
e_{i, k, s} & =\left(\begin{array}{l}
s \\
i
\end{array}\right) \prod_{h=i+1}^{s}(h k-1) \prod_{h=s-i+1}^{s}(h k+1), \\
f_{i, k, s} & =\left(\begin{array}{l}
s \\
i
\end{array}\right) \prod_{h=i+1}^{s}(h k+1) \prod_{h=s-i+1}^{s}(h k-1) .
\end{aligned}
$$

Proof. First we prove (6.21). From (6.19) we obtain

$$
\sum_{i=0}^{s} f_{i, k, s} t^{i}=\sum_{j=0}^{s} d_{j, k, s}(t-1)^{j} .
$$

Binomial theorem then implies that

$$
f_{i, k, s}=\sum_{j=i}^{s}\left(\begin{array}{l}
j \\
i
\end{array}\right)(-1)^{j-i} d_{j, k, s} .
$$

Thus equality (6.21) is equivalent to

$$
\left(\begin{array}{l}
s \\
i
\end{array}\right) \prod_{h=i+1}^{s}(h k+1) \prod_{h=s-i+1}^{s}(h k-1)=\sum_{j=i}^{s}\left(\left(\begin{array}{l}
j \\
i
\end{array}\right)(-1)^{j-i} \frac{(2 s-j) !}{j !(s-j) !} k^{s-j} \prod_{h=s-j+1}^{s}(h k-1)\right) .
$$

On both sides of (6.22) there are polynomials of degree $s$ in variable $k$. Therefore it suffices to prove the equality for every $k=\frac{1}{r}$ with $r \in \mathbb{N}_{1}, r \geq s+1$.

Lemma 7 implies

$$
\begin{aligned}
& 1=S_{5}(n=r, A=i, B=s) \\
&= \sum_{j=i}^{s} \frac{(2 s-j) !(r-s+j-1) !(s-i) !(r+i) !}{(j-i) !(s-j) ! s !(r+s) !(r-s+i-1) !} \\
&= \frac{\sum_{j=i}^{s}\left(\begin{array}{l}
j \\
i
\end{array}\right) \frac{(2 s-j) !}{j !(s-j) !} \frac{(r-s+j-1) !}{(r-s-1) !}}{\left(\begin{array}{l}
s \\
i
\end{array}\right) \frac{(r+s) !(r-s+i-1) !}{(r+i) !}} \\
&= \frac{\sum_{j=i}^{s}\left(\begin{array}{l}
j \\
i
\end{array}\right)(-1)^{j-i} \frac{(2 s-j) !}{j !(s-j) !} \prod_{h=s-j+1}^{s}(h-r)}{\left(\begin{array}{l}
s \\
i
\end{array}\right) \prod_{h=i+1}^{s}(h+r) \prod_{h=s-i+1}^{s}(h-r)} \\
& \sum_{j=i}^{s}\left(\begin{array}{l}
j \\
i
\end{array}\right)(-1)^{j-i} \frac{(2 s-j) !}{j !(s-j) !} k^{s-j} \prod_{h=s-j+1}^{s}(h k-1) \\
&\left(\begin{array}{l}
s \\
i
\end{array}\right) \prod_{h=i+1}^{s}(h k+1) \prod_{h=s-i+1}^{s}(h k-1)
\end{aligned}
$$

hence (6.22) and (6.21) follow.

Putting $k:=-k$ into (6.21) and applying binomial theorem in the same way we obtain (6.20). 


\subsection{Bounds}

By Lemma 14 the function $p_{k, s}$ is Padé approximation, hence we know its properties in the neighbourhood of 1. Here we find global bounds for $p_{k, s}$ that are necessary for functionality of our algorithm.

We need another two technical lemmas.

Lemma 16. For $k \in \mathbb{R}$ and $s, m \in \mathbb{N}_{1}$

$$
\sum_{i=0}^{s} \frac{(-1)^{s-i}(s+i) !}{(s-i) ! i !(s+m+i) !} \prod_{h=i+1}^{s}(h k-1) \prod_{h=s+m}^{s+m+i-1}(h k-1)=\frac{(s+m-1) !}{(2 s+m) !(m-1) !} \prod_{h=1}^{s}(h k+1) .
$$

Proof. On both sides there are polynomials of degree $s$ in variable $k$. Therefore it suffices to prove the equality for every $k=\frac{1}{r}$ with $r \in \mathbb{N}_{1}, r \geq 2 s+m$.

Lemma 9 implies

$$
\begin{aligned}
1 & =S_{7}(n=r, A=s, B=m) \\
& =\sum_{i=0}^{s} \frac{(-1)^{i}(s+i) !(2 s+m) !(m-1) !(r-i-1) !(r-s-m) ! r !}{(s-i) ! i !(s+m+i) !(s+m-1) !(r-s-1) !(r-s-m-i) !(r+s) !} \\
& =\sum_{i=0}^{s}\left(\frac{(-1)^{i}(s+i) !(2 s+m) !(m-1) !}{(s-i) ! i !(s+m+i) !(s+m-1) !} \prod_{h=i+1}^{s}(r-h) \prod_{h=s+m}^{s+m+i-1}(r-h) \prod_{h=1}^{s}(h+r)^{-1}\right) \\
& =\sum_{i=0}^{s}\left(\frac{(-1)^{s-i}(s+i) !(2 s+m) !(m-1) !}{(s-i) ! i !(s+m+i) !(s+m-1) !} \prod_{h=i+1}^{s}(h k-1) \prod_{h=s+m}^{s+m+i-1}(h k-1) \prod_{h=1}^{s}(h k+1)^{-1}\right) .
\end{aligned}
$$

This implies the result.

Lemma 17. For $k \in \mathbb{N}_{2}$ and $j \in \mathbb{N}_{1}$ with $j \geq 2 s+1$

$$
\sum_{i=0}^{s}(-1)^{s-i} d_{s-i, k, s}\left|g_{j-s+i, k}\right|>0 .
$$

Proof. Put $m:=j-2 s \geq 1$. Then

$$
\begin{aligned}
\text { LHS } & =\sum_{i=0}^{s}(-1)^{s-i} d_{s-i, k, s}\left|g_{j-s+i, k}\right| \\
& =\sum_{i=0}^{s}\left((-1)^{s-i} \frac{(s+i) !}{(s-i) ! i !} k^{i} \prod_{h=i+1}^{s}(h k-1) \frac{1}{k^{j-s+i}(j-s+i) !} \prod_{h=1}^{j-s+i-1}(h k-1)\right) \\
& =\frac{1}{k^{j-s}} \prod_{h=1}^{j-s-1}(h k-1) \sum_{i=0}^{s}\left(\frac{(-1)^{s-i}(s+i) !}{(s-i) ! i !(j-s+i) !} \prod_{h=i+1}^{s}(h k-1) \prod_{h=j-s}^{j-s+i-1}(h k-1)\right) \\
& =\frac{1}{k^{m+s}} \prod_{h=1}^{m+s-1}(h k-1) \sum_{i=0}^{s}\left(\frac{(-1)^{s-i}(s+i) !}{(s-i) ! i !(m+s+i) !} \prod_{h=i+1}^{s}(h k-1) \prod_{h=m+s}^{m+s+i-1}(h k-1)\right) .
\end{aligned}
$$

Lemma 16 implies

$$
\text { LHS }=\frac{1}{k^{m+s}} \frac{(s+m-1) !}{(2 s+m) !(m-1) !} \prod_{h=1}^{s}(h k+1) \prod_{h=1}^{m+s-1}(h k-1)>0 .
$$

Now we find lower and upper bounds for the function $p_{k, s}$.

Lemma 18. For $k \in \mathbb{N}_{2}, \quad s \in \mathbb{N}_{1}$ and $t \in(0,1)$ we have $p_{k, s}(t)>\sqrt[k]{t}$.

Proof. Put

$$
\sum_{j=0}^{\infty} \alpha_{j, k, s}(t-1)^{j}:=\left(\sum_{j=0}^{s} d_{j, k, s}(t-1)^{j}\right) \sqrt[k]{t}=\left(\sum_{i=0}^{s} d_{i, k, s}(t-1)^{i}\right)\left(\sum_{i=0}^{\infty} g_{i, k}(t-1)^{i}\right)
$$


Lemma 12 and Lemma 13 imply

$$
\sum_{j=0}^{2 s} \alpha_{j, k, s}(t-1)^{j}=\sum_{j=0}^{s} c_{j, k, s}(t-1)^{j} .
$$

From (6.23) we obtain for $j \geq 2 s+1$

$$
\alpha_{j, k, s}=\sum_{i=0}^{s} d_{s-i, k, s} g_{j-s+i, k} .
$$

We have $g_{j-s+i, k}=(-1)^{j-s+i-1}\left|g_{j-s+i, k}\right|$. Lemma 17 implies

$$
\sum_{j=2 s+1}^{\infty} \alpha_{j, k, s}(t-1)^{j}=-\sum_{j=2 s+1}^{\infty}\left(\sum_{i=0}^{s}(-1)^{s-i} d_{s-i, k, s}\left|g_{j-s+i, k}\right|\right)(1-t)^{j}<0 .
$$

From (6.23), (6.24) and (6.25) we obtain

$$
\left(\sum_{j=0}^{s} d_{j, k, s}(t-1)^{j}\right) \sqrt[k]{t}=\sum_{j=0}^{2 s} \alpha_{j, k, s}(t-1)^{j}+\sum_{j=2 s+1}^{\infty} \alpha_{j, k, s}(t-1)^{j}<\sum_{j=0}^{s} c_{j, k, s}(t-1)^{j} .
$$

Lemma 15 implies that

$$
\sum_{j=0}^{s} d_{j, k, s}(t-1)^{j}>0
$$

hence

$$
p_{k, s}(t)=\frac{\sum_{j=0}^{s} c_{j, k, s}(t-1)^{j}}{\sum_{j=0}^{s} d_{j, k, s}(t-1)^{s}}>\sqrt[k]{t} .
$$

Lemma 19. For every $k \in \mathbb{N}_{2}, \quad s \in \mathbb{N}_{1}$ and $x>1$ we have $p_{k, s}(t)>1$.

Proof. Directly from the definition of $c_{j, k, s}$ and $d_{j, k, s}$ we obtain

$$
p_{k, s}(t)=\frac{\sum_{j=0}^{s} c_{j, k, s}(t-1)^{j}}{\sum_{j=0}^{s} d_{j, k, s}(t-1)^{j}}=\frac{\sum_{j=0}^{s}\left(\frac{(2 s-j) !}{j !(s-j) !} k^{s-j} \prod_{h=s-j+1}^{s}(h k+1)\right)(t-1)^{j}}{\sum_{j=0}^{s}\left(\frac{(2 s-j) !}{j !(s-j) !} k^{s-j} \prod_{h=s-j+1}^{s}(h k-1)\right)(t-1)^{j}}>1 .
$$

The inequality is strict, since for $j \geq 1$ the main bracket in the numerator is greater than the main bracket in the denominator.

Now we prove that function $p_{k, s}(t)$ satisfies inequalities (3.2).

Lemma 20. For every $k \in \mathbb{N}_{2}, \quad s \in \mathbb{N}_{1}$ and $t \in \mathbb{R}^{+} \backslash\{1\}$

$$
\min (1, \sqrt[k]{t})<p_{k, s}(t)<\max (1, \sqrt[k]{t})
$$

Proof. The proof splits into four cases.

1) For $0<t<1$ Lemma 18 implies that $\sqrt[k]{t}<p_{k, s}(t)$.

2) Lemma 15 implies that $e_{j, k, s}=f_{s-j, k, s}$ for every $j, k, s$. Hence

$$
p_{k, s}\left(\frac{1}{t}\right)=\frac{1}{p_{k, s}(t)}
$$

Using this and the first case we obtain that for $t>1$ we have $p_{k, s}(t)<\sqrt[k]{t}$.

3) For $t>1$ Lemma 19 implies that $p_{k, s}(t)>1$.

4) Using this and (6.27) we obtain for $0<t<1$ that $p_{k, s}(t)<1$.

\section{Acknowledgements}

The author would like to thank to professor Andrzej Schinzel for recommendation of the paper [14] and also to 
author’s colleagues Kamil Brezina, Lukáš Novotný and Jan Štěpnička for checking the results. Publication of this paper was supported by grant P201/12/2351 of the Czech Science Foundation, by grant 01798/2011/RRC of the Moravian-Silesian region and by grant SGS08/PrF/2014 of the University of Ostrava.

\section{References}

[1] Householder, A.S. (1970) The Numerical Treatment of a Single Nonlinear Equation. McGraw-Hill, New York.

[2] Hardy, G.H., Littlewood, J.E. and Pólya, G. (1952) Inequalities. Cambridge University Press, Cambridge.

[3] Borwein, J.M. and Borwein, P.B. (1987) Pi and the AGM. John Wiley \& Sons, Hoboken.

[4] Gauss, C.F. (1866) Werke. Göttingen.

[5] Matkowski, J. (1999) Iterations of Mean-Type Mappings and Invariant Means. Annales Mathematicae Silesianae, 12, 211-226.

[6] Karatsuba, A. and Ofman, Yu. (1962) Umnozhenie mnogoznachnykh chisel na avtomatakh. Doklady Akademii nauk SSSR, 145, 293-294.

[7] Schönhage, A. and Strassen, V. (1971) Schnelle Multiplikation Großer Zahlen. Computing, 7, 281-292. http://dx.doi.org/10.1007/BF02242355

[8] Fürer, M. (2007) Faster Integer Multiplication. Proceedings of the 39th Annual ACM Symposium on Theory of Computing, San Diego, California, 11-13 June 2007, 55-67.

[9] Brent, R.P. (1975) Multiple-Precision Zero-Finding Methods and the Complexity of Elementary Function Evaluation. In: Traub, J.F., Ed., Analytic Computational Complexity, Academic Press, New York, 151-176.

[10] Knuth, D.E. (1998) The Art of Computer Programming. Volume 2: Seminumerical Algorithms. Addison-Wesley, Boston.

[11] Brezina, K. (2012) Smíšené Průměry. Master Thesis, University of Ostrava, Ostrava.

[12] Karatsuba, A. (1995) The Complexity of Computations. Proceedings of the Steklov Institute of Mathematics, 211, 169183.

[13] Pan, V.Ya. (1961) Nekotorye skhemy dlya vychisleniya znacheni polinomov s veshchestvennymi koeffitsientami. Problemy Kibernetiki, 5, 17-29.

[14] Wilf, H. and Zeilberger, D. (1990) Rational Functions Certify Combinatorial Identities. Journal of the American Mathematical Society, 3, 147-158. http://dx.doi.org/10.1090/S0894-0347-1990-1007910-7

[15] Jarník, V. (1984) Diferenciální počet 1. Academia, Praha. 
Scientific Research Publishing (SCIRP) is one of the largest Open Access journal publishers. It is currently publishing more than 200 open access, online, peer-reviewed journals covering a wide range of academic disciplines. SCIRP serves the worldwide academic communities and contributes to the progress and application of science with its publication.

Other selected journals from SCIRP are listed as below. Submit your manuscript to us via either submit@scirp.org or Online Submission Portal.
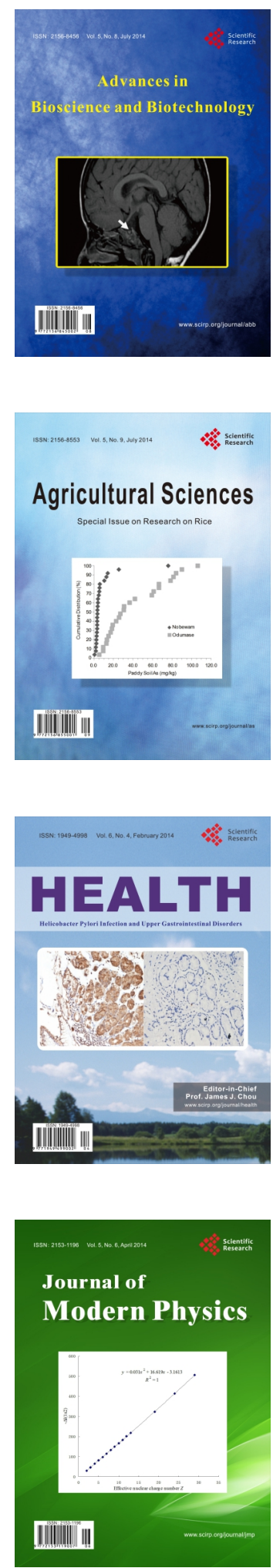
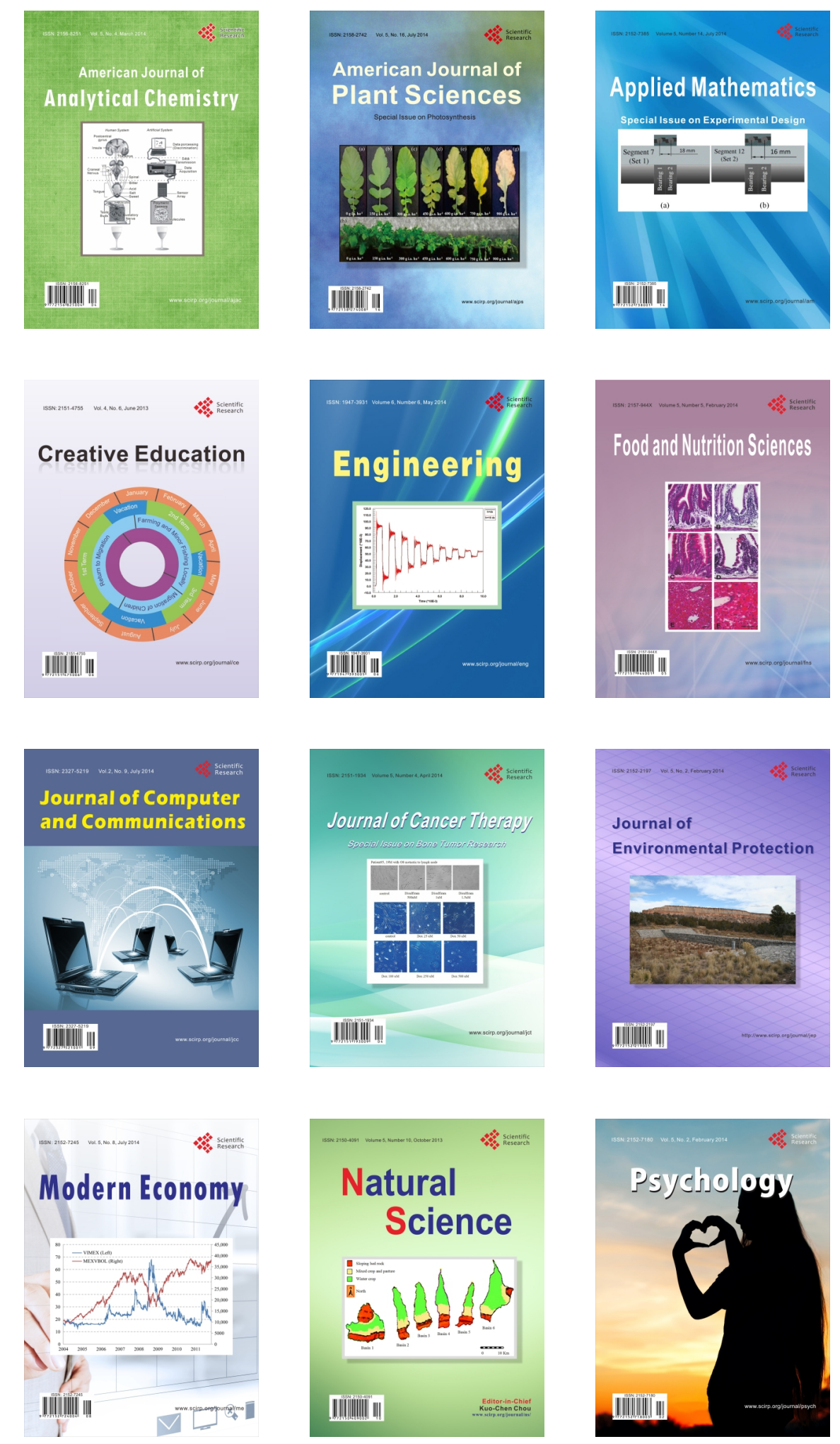